\title{
Human papillomavirus testing and the management of women with mildly abnormal cervical smears: an observational study
}

\author{
Gemma Rebello, Nick Hallam, George Smart, David Farquharson, Jane McCafferty
}

Following publication of a report in Health Technology Assessment the NHS is running a pilot scheme screening women for human papillomavirus if they have a mildly dyskaryotic or borderline smear. ${ }^{1}$ How reliable is testing for human papillomavirus as a marker for high grade disease in those with mildly abnormal smears?

\section{Participants, methods, and results}

Three hundred and thirty three consecutive new patients (aged 17 to 61 years, median 30 years) referred for colposcopy with persistent borderline or mildly dyskaryotic smears and who consented to the study were tested for human papillomavirus (high risk types only) with the Digene Hybrid Capture assay HC II (Abbott Laboratories, Maidenhead), by using cervical brush specimens placed in Digene transport medium, and were treated by large loop excision of the transformation zone.

The table summarises test performance, and table A on the BMJ's website presents results by age, smear history, and test cut off. ${ }^{2}$ Subjects aged under 30 years (166) were more likely than older subjects (167) to test positive for human papillomavirus $(79 \%(131 / 166) v$ $\left.45 \% \quad(75 / 167) ; \quad \chi^{2}=39.4, \quad \mathrm{df}=1, \quad \mathrm{P}<0.001\right) \quad$ and (dependent on this) to have cervical intraepithelial neoplasia grade 2 or 3 (high grade disease) (43\% (71/ 166) $\left.v 27 \%(45 / 167) ; \chi^{2}=8.5, \mathrm{df}=1, \mathrm{P}<0.01\right)$.

\section{Comment}

A proportion of patients with mildly abnormal smears will harbour high grade disease. ${ }^{3}$ Identifying this important clinical issue. Colposcopy itself does not identify high grade disease reliably and we asked whether testing for human papillomavirus might subgroup to target appropriate management is an improve diagnostic accuracy. We found that testing for human papillomavirus had a higher overall sensitivity (93\% [1 pg/ml cut off] to $85 \%$ [4 pg/ml]) than specificity $(55 \%[1 \mathrm{pg} / \mathrm{ml}]$ to $62 \%[4 \mathrm{pg} / \mathrm{ml}])$ in detecting those with high grade disease, thus limiting its usefulness as a surrogate marker (table). This differential was greater for younger women, for those with mild dyskaryosis, and at $1 \mathrm{pg} / \mathrm{ml}$ cut off (table). Testing performed best with negative predictive values (prevalence adjusted) of $96 \%$ in older women and $94 \%$ overall $(1 \mathrm{pg} / \mathrm{ml})$.

The report in Health Technology Assessment concludes that "the clearest role for HPV testing at the moment is in the management of women with borderline or mildly dyskaryotic smears. In particular, those aged above 30 years who test positive for high risk types could be referred immediately for colposcopy, while those younger than 30 years who test negative could receive less-intensive surveillance." The report supports limited introduction of testing for human papillomavirus, which should be carefully monitored, and encourages further research, including assessing the safety of returning to routine screening women with borderline or mild smears who test negative for human papillomavirus. Extrapolating our results to these guidelines would mean that $55 \%$ of those aged 30 years or over who test positive and are referred would have high grade disease but such disease might be missed in a small proportion of those testing negative and not referred (4\% for older subjects, $11 \%$ for younger ones), this being more significant for younger women if they then face less intensive surveillance. We recognise, however, that our study population, with repeated abnormal smears, differs from that at triage for a single abnormal smear when the prevalence of high grade disease would probably be lower.

Manos et al report that human papillomavirus testing is useful in triaging those with atypical squamous
Editorial by Manos

Colposcopy Clinic, Royal Infirmary of Edinburgh, Lothian University Hospitals NHS Trust, Edinburgh EH3 9YW

Gemma Rebello consultant cytopathologist George Smart consultant gynaecologist

David Farquharson patient services director, reproductive medicine

Jane McCafferty colposcopy sister

Regional Clinical Virology

Laboratory, City Hospital, Lothian University Hospitals NHS Trust,

Edinburgh

EH10 5SB

Nick Hallam consultant virologist

Correspondence to: N Hallam

BMJ 2001;322:893-4

Performance of human papillomavirus testing in detecting subjects with high grade disease according to age, smear history, and cut off value of test

\begin{tabular}{|c|c|c|c|c|c|}
\hline & \multirow{3}{*}{$\begin{array}{l}\text { Cut off value } \\
\text { of test }(\mathrm{pg} / \mathrm{ml})\end{array}$} & \multicolumn{4}{|c|}{ Test performance (\%) $(95 \% \mathrm{Cl})$} \\
\hline & & \multirow[b]{2}{*}{ Sensitivity } & \multirow[b]{2}{*}{ Specificity } & \multicolumn{2}{|c|}{ Predictive values* } \\
\hline & & & & Positive & Negative \\
\hline \multicolumn{6}{|l|}{ Age† } \\
\hline$<30$ yr $(n=166)$ & 1 & 94 (86 to 98 ) & 33 (24 to 43$)$ & 51 (43 to 60$)$ & 89 (74 to 96$)$ \\
\hline$\geqslant 30$ yr $(n=167)$ & 1 & 91 (79 to 97$)$ & 72 (64 to 79$)$ & 55 (43 to 65$)$ & 96 (89 to 98$)$ \\
\hline \multicolumn{6}{|l|}{ Smear history } \\
\hline Borderline $(n=75)$ & 1 & 86 (65 to 95) & 76 (63 to 85$)$ & 58 (41 to 74$)$ & 93 (82 to 98$)$ \\
\hline Mild dyskaryosis $(n=117)$ & 1 & 94 (84 to 98$)$ & 39 (29 to 52$)$ & 55 (44 to 65$)$ & 90 (74 to 96$)$ \\
\hline Both types $(n=141)$ & 1 & 95 (85 to 99) & 54 (44 to 63) & 48 (38 to 59$)$ & 96 (88 to 99$)$ \\
\hline \multirow[t]{3}{*}{ All $(n=333)$} & 1 & 93 (87 to 97$)$ & 55 (48 to 61$)$ & 52 (46 to 59$)$ & 94 (88 to 97$)$ \\
\hline & 2 & 91 (85 to 95) & 57 (51 to 64) & 53 (46 to 60$)$ & 93 (87 to 96$)$ \\
\hline & 4 & 85 (78 to 91$)$ & 62 (55 to 68 ) & 54 (47 to 62) & 89 (83 to 93$)$ \\
\hline
\end{tabular}

${ }^{*}$ Adjusted for prevalence of high grade disease.

†That there were almost exactly the same number of subjects in each of the two age groups was coincidental. 
cells of undetermined significance (approximates to borderline change $)^{4}$; others are evaluating this but believe that testing has limited potential in triaging low grade squamous intraepithelial lesions (approximates to mild dyskaryosis). ${ }^{5}$ We found an overall test sensitivity and specificity of $86 \%$ and $76 \%$ for 75 subjects with borderline smears, $94 \%$ and $39 \%$ for 117 with mild dyskaryosis, and $95 \%$ and $54 \%$ for 141 with both smear types $(1 \mathrm{pg} / \mathrm{ml})$. We welcome the NHS pilot scheme while advising caution in the clinical use of testing for human papillomavirus, especially at a single point in time.

We thank nursing and medical colleagues at the colposcopy clinic for their assistance.

Contributors: GR had the idea for the study and reviewed the histology. NH performed human papillomavirus testing, wrote the paper, and will act as guarantor. All the authors were clinically involved in carrying out the study and contributed to the final version of the paper.
Funding: The Royal Infirmary of Edinburgh Endowment Fund. Abbott Laboratories kindly provided human papillomavirus testing kits at a discount.

Competing interests: GR and NH have been sponsored by Digene Diagnostics to attend several conferences.

1 Cuzick J, Sasieni P, Davies P, Adams J, Normand C, Frater A, et al. A systematic review of the role of human papillomavirus testing within a cervical screening programme. Health Technol Assess 1999;3 (14)

2 Cuzick J, Beverley E, Ho L, Terry G, Sapper H, Mielzynska I, et al. HPV testing in primary screening of older women. Br J Cancer 1999:81:554-8. E. Where's the high-grade che minimally abnormal Papanicolaou diagnoses. Obstet Gynecol 1998;91:973-6.

4 Manos MM, Kinney WK, Hurley LB, Sherman ME, Shieh-Ngai J, Kurman $\mathrm{RJ}$, et al. Identifying women with cervical neoplasia: using human papillomavirus DNA testing for equivocal Papanicolaou results. JAMA 1999;281:1605-10.

5 The Atypical Squamous Cells of Undetermined Significance/Low-Grade Squamous Intraepithelial Lesions Triage Study (ALTS) Group. Human papillomavirus testing for triage of women with cytologic evidence of low-grade squamous intraepithelial lesions: baseline data from a randomized trial. J Natl Cancer Inst 2000;92:397-402.

(Accepted 11 October 2000)

\title{
Do obstetric complications explain high caesarean section rates among women over 30 ? A retrospective analysis
}

\author{
Jacqueline S Bell, Doris M Campbell, Wendy J Graham, Gillian C Penney, Mandy Ryan, Marion H Hall
}

Dugald Baird

Centre for Research in Women's Health,

Department of

Obstetrics and

Gynaecology,

Aberdeen Maternity

Hospital, Aberdeen

AB25 2ZL

Jacqueline S Bell

research fellow

Doris M Campbell

senior lecturer

Wendy J Graham

professor

Gillian C Penney

senior lecturer

Marion H Hall

consultant

obstetrician

Health Economics

Research Unit,

University of

Aberdeen,

Aberdeen

AB25 2ZD

Mandy Ryan

Medical Research

Council senior fellow

Correspondence to:

J S Bell

j.bell@abdn.ac.uk

BMJ 2001;322:894-5

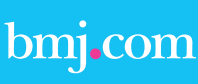

Figure showing odds ratios for delivery by

caesarean section

in women with no

history of such

sections appears on

the BMJ's website
As a growing proportion of women delay childbearing into their later reproductive years, the risks and costs associated with advancing maternal age become increasingly important. Extensive evidence shows that both obstetric interventions and obstetric complications are more common among older women, ${ }^{1}$ and it is often assumed that the interventions are a consequence of the complications. Delivery by caesarean section is one such intervention that is associated with maternal age and is of importance for public health. The extent to which the association is explained by obstetric complications is, however, not known. Martel et al showed that an association between maternal age and rates for primary caesarean section persisted after adjustment for induction of labour, epidural anaesthesia, meconium stained amniotic fluid, and fetal distress. ${ }^{2}$ We aimed to build on this finding by considering a greater number of obstetric complications in a much larger population and with more detailed records.

\section{Methods and results}

We obtained our information from the Aberdeen Maternal and Neonatal Databank for all singleton deliveries to city residents aged at least 20 years during 1988-97; totalling 23806 deliveries. ${ }^{3}$ We used logistic regression to obtain crude odds ratios for delivery by caesarean section among older women (age categories 30-31, 32-33, 34-35, 36-37, 38-39, and 40 and over) compared with a reference group of women aged 20-29 years. Primiparous and multiparous women were analysed separately, as were elective and emergency caesarean sections. We investigated the potential confounders of the association between age and outcome, and we also checked for any evidence of effect modification with the same variables-maternal sociodemographic characteristics and obstetric history.
We selected the obstetric complications and interventions associated with a higher probability of caesarean section, which might explain the association with age (see figure on web). Using multivariate logistic regression we adjusted the crude odds ratios for these variables, also controlling for any identified confounders and stratifying by effect modifiers.

The association between maternal age and caesarean section varied depending on how the baby presented at delivery and whether a woman had previously had a caesarean. Among women who had not previously had a caesarean section and whose babies presented normally at delivery there was a strong and consistent relation between maternal age and delivery by caesarean section that remained after controlling for relevant obstetric complications and identified confounders (see figure on web). Among women who had had a previous caesarean section or whose babies presented abnormally the association between maternal age and both elective and emergency sections was greatly reduced (results not shown).

\section{Comment}

The observed relation between maternal age and caesarean section cannot be explained by the obstetric complications we considered. They add to previous findings by including greater numbers of obstetric complications and deliveries in the analysis. This raises the question of why rates for caesarean section are high among older mothers, and whether they may be explained by physiological or other factors we have been unable to control for. Medical causes that have been suggested include reduced uterine function and pelvic compliance among older women. ${ }^{4}$ However we expected that length of labour would act as a proxy for these factors in our analysis. Many authors have identi- 